\title{
EI hecho protagónico de la apropiación del suelo en la forma urbana hacia la ciudad compacta. Caso de estudio: Zonas Metropolitanas de Córdoba y Guadalajara.
}

\section{Lilly Areli Sánchez Correa ${ }^{1}$, Guadalupe Noemi Uehara Guerrero²}

Facultad de Arquitectura, Universidad Veracruzana. Veracruz, México

E-mail: 1arsanchez@uv.mx, ${ }^{2}$ guehara@uv.mx

\begin{abstract}
Resumen. Entender la dinámica de la ciudad a través del comportamiento de las formas de apropiación del suelo muestra una directriz relevante en las tendencias de expansión y consolidación que manifiesta la ciudad. Es en este escenario que el ejido mexicano, se inserta protagónicamente en la geografia de la ciudad, ahora conduciendo crecimientos, y hasta nuevas formas urbanas. La ciudad cambia de ser una entidad difusa, plana y extendida a otra geografía, con nuevas formas, como islas urbanas, células en un mar de espacios intersticiales, archipiélagos, y otras semejanzas de espacios desvinculados que muestran otra condición morfológica.

El estudio que se presenta tomando referentes en las zonas metropolitanas en Veracruz y Jalisco, evidencia dos etapas: la coexistencia de la ciudad con este entorno que favoreció la venta y reventa bajo esquemas ilegales, en espera de la regularización; y posteriormente, en un cambio estructural dictaminado en las leyes agrarias, el efecto trasciende a la ciudad, al gozarse de una gran oferta de suelo para la inversión inmobiliaria, haciendo una ciudad de bloques separados entre si conforme al mercado de suelo y la voluntad de la población ejidataria, lo cual dista de un adecuado ordenamiento.
\end{abstract}

Palabras clave: eijido, forma urbana, expansión.

\section{Un ejido a la mexicana}

La expresión generada a partir de las formas de apropiación del suelo según su régimen de propiedad es una de las lecturas que dan respuesta con mayor sustento a las causas de la configuración urbana. En este sentido, se considera pertinente entender en principio este concepto de pertenencia del suelo ejidal a partir de su propósito inicial. Un ejido a la mexicana se hace necesario identificarlo así, en función de revisar brevemente el fundamento que le da origen, en razón de que este tipo de tenencia hace acto de presencia en la distribución de la propiedad en el país, atendiendo a la demanda postrevolucionaria de principios del siglo $\mathrm{XX}$, la cual exigía la restitución y dotación de tierras para una masa campesina que había sido despojada de sus bienes en aras de un acaparamiento de tierras propiciado por las prácticas latifundistas de periodos precedentes decimonónicos, impulsores de la propiedad privada.

En la reminiscencia de una figura antecedente identificada como ejido en la época virreinal, esta tenencia del suelo que se instaura en 1917 rescata aquellas cualidades que habían sido observadas con anterioridad, sin tratarse precisamente del mismo tipo de propiedad. El ejido anterior -proveniente del derecho romano- se estipulaba para fines recreativos y de pastoreo, así como para el ensanche de las ciudades, con tres cualidades destacables: ser inalienable, ser imprescriptible y ser previsto en devolución a la Corona Española en caso de no cumplir con su cometido ${ }^{1}$.

Con las variantes aplicables, el ejido mexicano siglo $\mathrm{XX}$, retoma tal caracterización 
en el sentido de ser un bien fuera del mercado y no generador de derechos, de acuerdo al Constituyente de 1917, además de ser restituible en este caso a la Nación, la cual al ostentar la propiedad originaria podría disponer de él para fines de utilidad pública, sometiéndose a procesos de expropiación e indemnización, de acuerdo a lo estipulado posteriormente en el Código Agrario de 1934².

Es esta forma de propiedad la que permanentemente fue abordada bajo los lineamientos agrarios pensando poco -o quizás nada-, en su coexistencia urbana. Al analizar los tipos de tenencia de la tierra, sobre todo vinculados a la experiencia de la ciudad, habrán de considerarse dos factores: su origen y su ocupación; el origen referido al tipo de propiedad privada o ejidal, y su ocupación, en cuanto al tipo de utilización, legal o ilegal. Al respecto, la relación no es simplemente dual. Parecería que la propiedad privada es legal y la ejidal, ilegal, y no es así; dado que la complejidad urbana ha adquirido matices distintos. Si bien la propiedad privada preferentemente es legal, existen también en la ciudad, maneras de adquirir suelo y ocuparse fuera de las disposiciones legales y reglamentarias urbanas lo que la convierte en ilegal, en espera de su regularización, ya sea estatal, como en el estado de Veracruz; o municipal, como en el estado de Jalisco, atendiendo a las legislaciones respectivas.

En el caso del ejido, en su convivencia con la ciudad, a excepción de tratarse de una zona urbana ejidal adyacente, su ocupación siempre fue ilegal, dada la posición de esta tenencia como un bien nacional fuera de mercado; es posible actualmente ubicarlo en una situación de ocupación legal, atendiendo a su origen, pero habiendo cursado el proceso de desincorporación del régimen y obtención de su dominio pleno.

\section{La ilegalidad periférica regularizada}

Cuando expertos en el tema como Durand y Gilbert $^{3}$ abordaron el estudio de la invasión al ejido por parte de la ciudad y la caracterización de los asentamientos humanos irregulares, en relación a un hacer o no hacer del Estado, la evidencia de un desbordamiento de la ciudad sobre áreas circundantes de régimen de propiedad distinta era ya un hecho. El total de ciudades mexicanas experimentaban este fenómeno; un poco de expansión hacia zonas de tenencia privada y una gran periferia en su totalidad tapizada de ejidos que iniciaron un mercado ilegal. Se trataba de una oferta de terrenos baratos para una población requirente de un espacio donde habitar y que compraba en "ejido" por no tener capacidad económica para otra opción, con el conocimiento de la ausencia de seguridad legal a la que se enfrentaba, pero con una esperanza que después se volvió proceso reiterado de adquirir ilegalmente y regularizar después.

"La urbanización ilegal lleva implícita una oferta de suelo barato, lo que puede interpretarse como un encuentro afortunado entre las características de la oferta y el perfil de la demanda: la anchura de la ciudad autoproducida coincide con la estrechez de los recursos de la mayor parte de la población"4.

La población que enfrenta estos procesos es de origen rural, proveniente de un campo con desabastos y muchas veces deprimido que obliga a tratar de encontrar mejores condiciones de habitabilidad en la ciudad. A ello se aúna la población que proviene del centro, cuyo desplazamiento es obligado por los altos costos generados de vivienda en renta y por procesos de gentrificación.

"Además de la migración campo ciudad, se generan movimientos del centro a la periferia, así como dentro de esta última, lo cual produce una masa de población en situación de ciudadanía de segunda o subciudadanía, a la que se le han limitado sus derechos a la vivienda y a la ciudad".

El ejido es un concepto que deja claro su carácter al identificar que su área acreditada no está en el mercado; como lo señalaba el artículo 52 de la Ley de la Reforma Agraria, refiriéndose a las cualidades de su origen. No obstante, en los entornos urbanos, el Estado Mexicano toma la decisión de reconocer las formas ilegales como un problema público ${ }^{6}$. Así las cosas, la venta ilegal es consentida en tanto que funciona como válvula de escape para atender el requerimiento de suelo de una masa de población carente de posibilidades de adquirir suelo en propiedad privada. Por 
consiguiente, la regularización se constituye en una causa de utilidad pública, por lo que sustituyendo la sanción que se le impusiera al ejidatario por la venta ilícita, lo inserta en un proceso de regularización, en el que la relación expropiación - indemnización resulta gravosa para el adquirente del suelo y beneficiosa para el ejidatario, quien recibe un pago adicional además del obtenido por la operación precedente.

La Zona Metropolitana de Guadalajara es un ejemplo evidente, así como las Zonas Metropolitanas en el estado de Veracruz, en las que la presencia de zonas ejidales en el entorno de la ciudad sobre municipios colindantes preferentemente, tejieron un armado de ciudad que dista de contar con los principios básicos de estructura vial, tramas urbanas, dotación de equipamiento suficiente, entre otras cosas. La zona metropolitana de Guadalajara cuenta con 58 ejidos en los cuatro municipios centrales, de los cuales se eligió a manera de muestra el Municipio de Tonalá, el cual cuenta con 9 ejidos y está ubicado al Este de Guadalajara, con mayor participación de precariedad respecto del total de la zona periférica ${ }^{7}$. Este municipio recibe la expansión de las zonas menos favorecidas del Oriente de Guadalajara; en franja posición contrapuesta al Oeste de la ciudad, la cual ha encontrado en Zapopan, el área de expansión de mayor plusvalía en la Zona Metropolitana.

Con bases en datos registrales, se obtiene que en el período 1969 - 1995 se tramitó la regularización de 23 colonias en 262 has. Para 19991, la Corett ya había regularizado 10,000 predios sobre 19 colonias en los Ejidos San José Tateposco, Zalatitán y El Rosario ${ }^{8}$; en tanto que el espacio de mayor relevancia por su regularización fue la Colonia Jalisco, integrada por 200 manzanas y ubicada en el Ejido Zalatitán9.

Esta forma de ocupación del espacio periférico, en su mayoría perteneciente al régimen ejidal desde la década de 1960 , de acuerdo con Salazar ${ }^{10}$ generó la desaparición de la actividad agrícola y un impacto ambiental; no obstante, es de reconocerse que cuando menos propició un crecimiento continuo, no disperso y poroso como el que se ha dado en el presente siglo, a través de islas y espacios intersticiales. $\mathrm{Al}$ respecto, es viable observar en estas zonas regularizadas y consolidadas una continuidad, posiblemente inadecuada, más sin embargo próxima de la ciudad, facilitando posibles ordenamientos urbanos.

\section{El mercado ejidal en la expansión difusa.}

La secuencia sintetizada por Jiménez y Ayala $^{11}$ muestra el proceso experimentado en la ocupación del suelo: de hacendados a ejidatarios y de éstos a ocupantes irregulares y ahora a desarrolladores inmobiliarios. En este último paso, la ciudad modifica su rumbo de una expansión en la ilegalidad para incorporarse a un mercado formal de promoción inmobiliaria, produciendo otra forma de ciudad.

En 1992 se genera la reforma constitucional en materia agraria, reconociendo como propietarios a los sujetos de derecho agrario, por lo que se reitera nuevamente la mínima alusión que se hace de la ciudad. Al respecto, el artículo 9 de la Ley Agraria, reglamentaria de artículo 27 constitucional señala puntualmente: "Los núcleos de población ejidales o ejidos tienen personalidad jurídica $y$ patrimonio propio y son propietarios de las tierras que les han sido dotadas...."12. Esta tajante disposición hizo creer a la población beneficiada que al instante había ocurrido el cambio de tal régimen de propiedad. El proceso reglamentado fue explicado a la población para finalmente obtener el dominio pleno de sus parcelas, transitando desde la delimitación de áreas y la certificación parcelaria hasta obtener la titularidad de la propiedad.

Asimismo, era necesario aclarar que el cambio de régimen de propiedad de parcelas anteriormente agrícolas no las hacía al momento urbanas, sino que ello dependería de su localización en los programas de ordenamiento urbano, distinguiendo su posición dentro de áreas urbanizables a diferencia de aquellas que pudieran no serlo. Al respecto, era importante fijar la atención en el Artículo 2 de la citada Ley, en el que se estipula que toda acción motivada por ejercer el derecho de propiedad que se relacione con el aprovechamiento urbano deberá sujetarse a lo dispuesto en la Ley General de Asentamientos Humanos. $\mathrm{Si}$ obtener el dominio pleno de las parcelas 


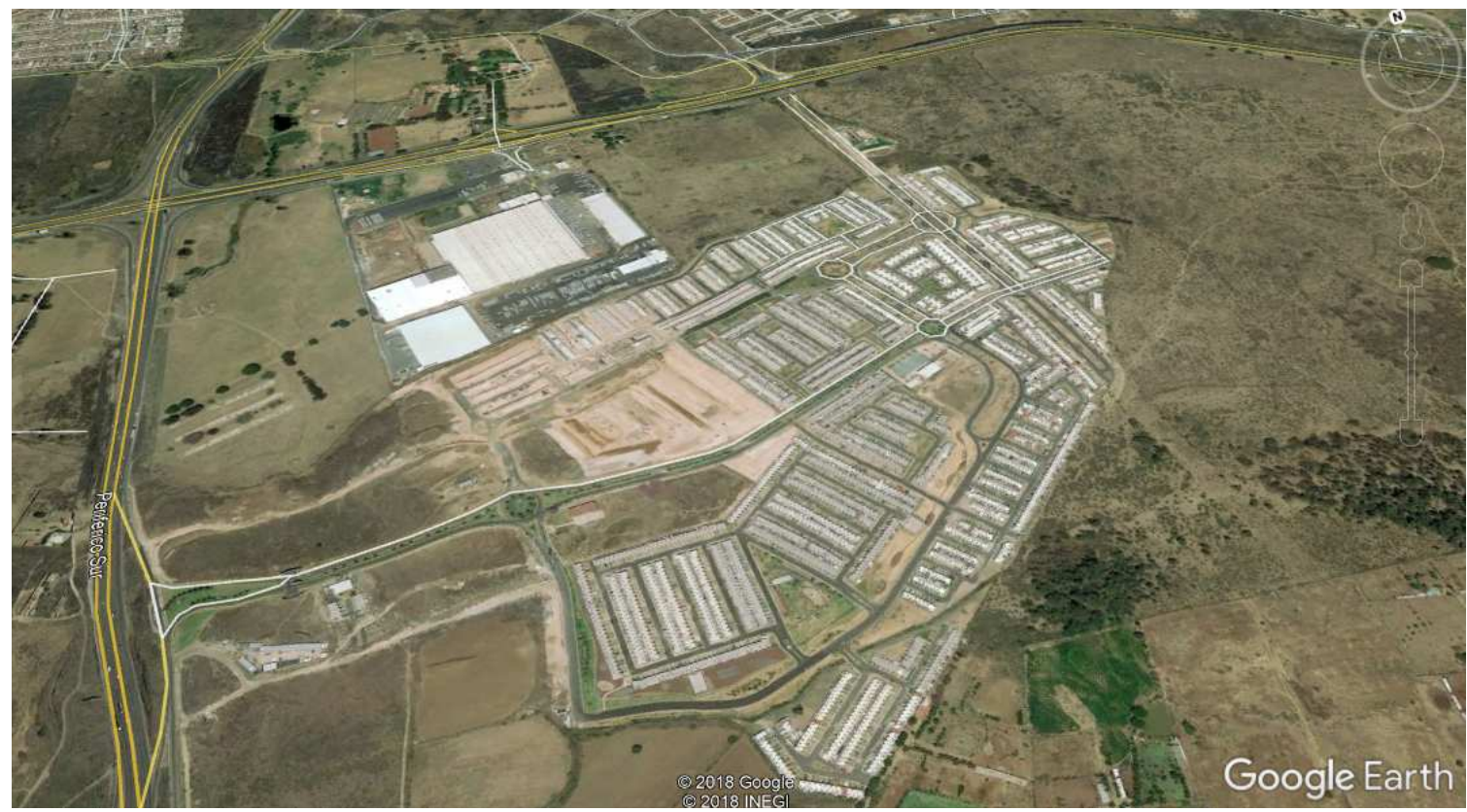

Fig. 1. Fraccionamiento Colinas de Tonalá en parcelas ejidales. (Fuente: Google Earth,2018)

por los ejidatarios implicaba sujetarse a una regulación urbana, quizás pensarían que era mejor el sistema anterior por el que se vendían lotes sin servicios y se esperaba una indemnización. En ese razonamiento, algunos núcleos ejidales optaron o no por la obtención del dominio pleno. La otra opción que apareció fue la presencia de un fuerte sector inmobiliario privado requirente de suelo urbanizable que anteriormente estaba atrapado por la barrera del régimen ejidal. De esta manera, resultó más atractivo vender las parcelas al inversionista como comprador más seguro que la venta al menudeo por lotes ${ }^{13}$.

El sector inmobiliario es uno de los productores mas destacados en la producción del espacio urbano, dada la construcción masiva de viviendas con las políticas públicas

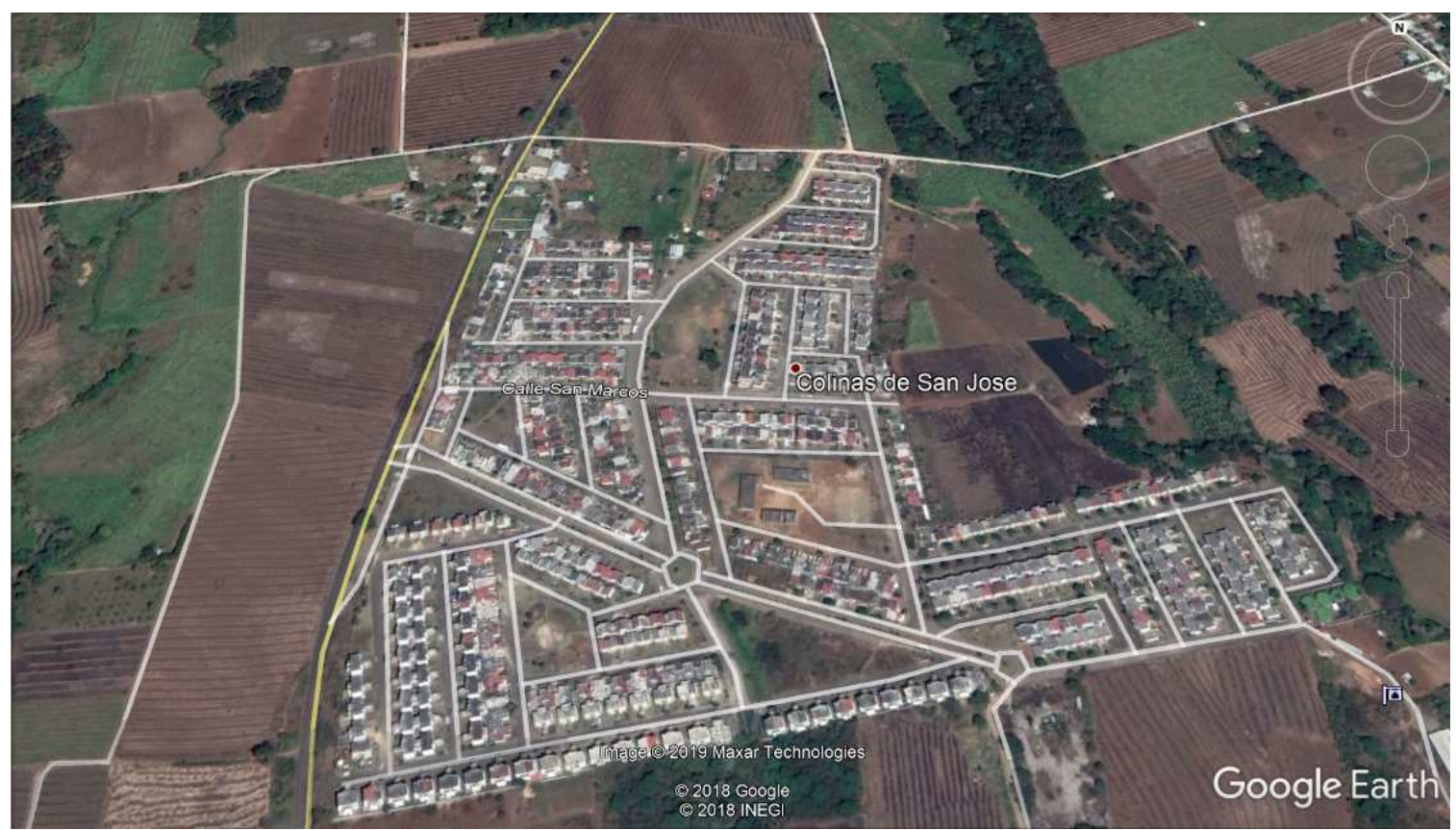

Fig. 2. Fraccionamiento Colinas de San José en parcelas ejidales (Fuente: Google Earth,2018) 
y crediticias que le son favorables ${ }^{14}$. El inicio del presente siglo marcó las pautas normativas para acrecentar una ciudad difusa y fragmentada; habría que cumplir un máximo número de viviendas, delegando la ubicación.

Derivado de ello, se crearon fraccionamientos residenciales distante de la ciudad, adquiridos por la venta de parcelas de ejidatarios que "decidieron vender". Si la oferta de suelo anteriormente ejidal, estuviere más próxima al área urbanizada habría menos dispersión. La afirmación expuesta apunta al hecho de que tanto promotores privados inmobiliarios como ejidatarios en su voluntad de vender parcelas han coadyuvado a esta ciudad resultante en archipiélago, carente de conexiones entre las partes y con altos costos de subsistencia para sus habitantes. Además de la lejanía es observable como las figuras parcelarias subsisten en los desarrollos de los fraccionamientos tal cual como fue la voluntad del ejidatario vendedor.

Un análisis comparativo entre las zonas metropolitanas de Córdoba, Veracruz y Guadalajara, Jalisco permiten visualizar el problema a nivel nacional. Las constantes son: Desarrollos masivos superiores a 50 has que implicaron la compra de varias parcelas de acuerdo a oferta del ejidatario o conveniencia del comprador, falta de continuidad urbana, trazo o trama acomodada a la figura parcelaria y no a los requerimientos de fluidez vial, altos costos de movilidad y generación de espacios intersticiales posibles de ocuparse para lograr la integración de los desarrollos hacia la compactación de la ciudad.

\section{Conclusiones. Un aporte hacia la ciudad compacta.}

En síntesis, a cien años de experiencia ejidal mexicana, resulta relevante para el estudio urbanístico comprender las etapas sucesivas por las que este tipo de propiedad ha incidido en las configuraciones urbanas, identificando causas $\mathrm{y}$ repercusiones que finalmente han incidido en esta forma de hacer ciudad. Las características del ejido son observables desde su origen para comprender causas y motivaciones, se trataba entonces de una población campesina requirente de suelo insertada en el ámbito rural, siendo hasta la década de 1960 cuando inicia la generación de fricciones en una coexistencia no favorable a los requerimientos del orden urbano.

Es posible al respecto identificar cuatro etapas de desarrollo. La primera etapa caracterizada por una estabilidad urbana con un ámbito agrario distante y preeminente en su atención, tanto por la legislación como por las políticas públicas dedicadas fehacientemente al bienestar rural; la segunda etapa, generada a partir de la expansión incontrolada de la ciudad sobre las áreas ejidales circundantes que propiciaron la sobreposición de áreas urbanas en una tenencia de suelo equivocada, lo cual derivó en una forma de hacer ciudad distante a los principios de diseño urbano que tímidamente empezaban a tratarse en la legislación urbana a partir de la década de 1970. Se estaba ya ante una ciudad diseñada por la población ejidal cuya única finalidad

Tabla 1. Comparativo de creación de desarrollos inmobiliarios similares en las zonas metropolitanas de Guadalajara Jalisco y Córdoba, Veracruz.

\begin{tabular}{|c|c|c|c|c|}
\hline AÑO & FRACCIONAMIENTO & PROPIETARIA & PARCELA & $\begin{array}{c}\text { SUPERFICIE } \\
\text { Ha }\end{array}$ \\
\hline \multirow{2}{*}{2007} & COLINAS DE TONALA & DOMUS & $6,7,8,9,10$, & $57-59-63.04$ \\
& EJIDO TONALA & DESARROLLADORA, & $16,17 \mathrm{Y} 18 \mathrm{Z}-1$ & \\
& MPIO. TONALA & S.A. DE C.V. & & \\
& Z.M. GUADALAJARA, JAL. & & & \\
& & & & $51 / 1-25-24.80$ \\
\hline 2009 & COLINAS DE SAN JOSE, & PROYECTOS & $9,15,18,19,20$, & \\
& EJIDO SAN JOSE DE & INMOBILIARIOS DE & $29,30,31,38$, & \\
& TAPIA & CULIACAN, S.A. DE & $40,41,43,45$, & \\
& MPIO. CORDOBA & C.V. & $46,49 \mathrm{Y} 51 \mathrm{Z}-1$ & \\
& Z.M. CÓRDOBA, VER. & & P1/1 & \\
\hline
\end{tabular}


era solventar su subsistencia económica y un Estado que dejaba hacer como válvula de escape para atender a la población requirente se suelo; de ahí el inicio de los procesos de regularización que llegaron a ser una práctica ya conocida de generar ventas ilegales para su posterior regularización.

Una tercera etapa surge por cambios estructurales en la política agraria al desear nuevamente capitalizar el suelo, como en etapas anteriores al origen del ejido, lo cual en el ámbito urbano abrió la posibilidad de comercializar el ejido ante mejores postores, abandonando la población ejidataria a la población de escasos recursos para realizar sus ventas a los inversionistas inmobiliarios. Al respecto, aun cuando el propósito era -según el discurso institucional- favorecer a la población ejidal haciéndola sujeta de créditos y participante de sociedades mercantiles, lo cierto fue que se abrió la puerta al mercado inmobiliario, agregándose los intereses ejidales a la generación de una ciudad que se expande según la mejor oferta parcelaria, en detrimento del orden urbano.

En propuesta a manera de conclusión, se plantea la viabilidad de una cuarta etapa a iniciar con base en la legislación expedida en 2016, misma que a través de reagrupaciones parcelarias indistintamente del origen de la propiedad sea por sí privada o desincorporada del régimen ejidal, pudiera revertir la expansión incontrolada hacia la compactación de las ciudades mediante la ocupación de espacios intersticiales que quedaron como producto de las decisiones voluntarias de las ventas ejidales.

Si bien es cierto que la inversión de empresas inmobiliaria en conjunto con las disposiciones estatales, más inscritas en el ámbito financiero que en el urbano propiciaron esta formas de creación de islas o células residenciales con amplios vacíos entre ellas, también es cierto que la oferta de suelo ejidal a la voluntad de sus acreditados coadyuvó a esta forma de ciudad, dependiendo de la disponibilidad de parcelas que hubiere en el mercado, siendo protagónica su inserción en el crecimiento urbano, lo cual dista de una manera congruente de generar una ciudad de mejor habitabilidad.

\section{Agradecimientos}

Al Mtro Arq. Héctor Saul Castillo Moyado Jefe de Asentamientos Humanos del H. Ayuntamiento de Córdoba. Período 2015 2018. Arq. Ivan Betancourt Morales, Director de Desarrollo Urbano. Gobierno de Tonalá, Jal. Arq. Irving Gerardo Monroy Ramírez de Arellano, Director de Regularización, Gobierno de Tonalá, Jal. y Dra. Edith Jiménez Huerta, Directora de División de Diseño y Proyectos Cultural del CUAAD, Universidad de Guadalajara.

\section{Notas}

1 Martínez Báez, 1931 y Soto, 2012.

2 Riveros, 2007.

3 Durand, 1983 y Gilbert, 1987.

4 Cabrales, 2000:70.

5 Urquidiz, 2013.

6 Salazar, 2018.

7 Jiménez y Ayala, 2015.

8 Rentería, 2000.

9 Cabrales, op.cit.

10 Salazar, 2010.

11 Jiménez y Ayala, op.cit.

12 Ley Agraria, 1992.

13 Pradilla, 2011.

14 Aguila, 2016.

\section{Referencias}

Aguila, José Luis. 2011. Espacio intersticial. Surgimiento y transformación. Caso: Tonalá, Jalisco en México. México: Universidad de Guadalajara - Universidad Internacional de Andalucía. 
Cabrales, Luis Felipe. 2000. "Proceso de metropolización y segregación social tonalteca." En Núñez, Beatriz (Comp.) Tonalá: una aproximación a su estudio, 6585. México: El Colegio de Jalisco.

Durand, Jorge. 1983. La ciudad invade al ejido. Proletarización, urbanización y lucha política en el Cerro del Judío, D.F. México: Ediciones de la Casa Chata No. 17.

Gilbert, Alany Peter Ward. 1987.Asentamientos populares versus poder del Estado: tres casos latinoamericanos: Ciudad de México, Bogotá y Valencia. México: Gustavo Gili, Serie: Arquitectura Latinoamericana.

Jiménez, Edith y María Ayala. 2015. "Los ejidos y comunidades indígenas ante la expansion de Guadalajara 1920 - 2000" En Olivera, Guillermo (Coord.) La urbanización social y privada del ejido. Ensayos sobre la dualidad del desarrollo urbano en México. 103-147. México: Universidad Nacional Autónoma de México. Estudios regionales.

--Ley Agraria. Diario Oficial de la Federación, 1992. http://www.diputados.gob.mx/ LeyesBiblio/pdf/13_250618.pdf

Martínez, Alberto. 1931. "El ejido en la legislación de la época colonial”. Revista Universidad de México 8. 112-117. .

Pradilla, Emilio. 2011. "Zona Metropolitana del Valle de México: una ciudad baja, dispersa, porosa y de poca densidad." En Pradilla, Emilio (Comp.) Ciudades compactas, dispersas, fragmentadas.. 257-293. México: Universidad Autónoma Metropolitana Xochimilco.

Rentería, Javier. 2000. “De la metropolización a la desigualdad espacial: El caso de Tonalá. Un municipio conurbado." En Nuñez, Beatriz (Comp.) Tonalá: una aproximación a su estudio. México: El Colegio de Jalisco.

Riveros, Leonardo. 2007. La expropiación y los terrenos ejidales y comunales". Estudios agrarios. Revista de la Procuraduría Agraria 33 www.pa.gob.mx.

Salazar, Clara. 2018. "Transformación institucional y gobierno de los asentamientos informales". En Le Galés, Patrick y Vicente Ugalde (Edit.) Gobernando la ciudad de México. Lo que se gobierna u lo que no se gobierna en una gran metrópoli . México: El Colegio de México.
Soto Alva, Enrique. 2012. "El suelo de origen social". En Quiroz Rothe, Héctor y Esther Maya Pérez. (Comp) Urbanismo. Temas y tendencias. México: Universidad Nacional Autónoma de México.

Urquidiz, Octavio. 2013. “Anverso y reverso ¿Planeación vs. Gestión metropolitana?”. En Urquidiz, Octavio, Cabrales, José Luis et al (Comp) Metrópolis en movimiento. México: Colegio de Jalisco. 